\title{
Desbaste seletivo em teste de procedências e progênies de Astronium fraxinifolium Schott com base na variabilidade genética
}

\author{
Selective thinning in provenance and progeny test of \\ Astronium fraxinifolium Schott based on genetic variability \\ Maiara Ribeiro Cornacini' ${ }^{1}$ Janaína Rodrigues da Silva ${ }^{1}$, \\ Kelly Cristina da Luz' ${ }^{1}$ José Cambuim², Wanderley dos Santos ${ }^{1}$, \\ Mário Luiz Teixeira de Moraes ${ }^{3}$ e Ananda Virginia de Aguiar ${ }^{4}$
}

\begin{abstract}
Resumo
O objetivo do trabalho foi propor um desbaste seletivo visando o manejo de um teste de procedências e progênies de Astronium fraxinifolium Schott com base no conhecimento da variabilidade genética, visando a conservação do seu potencial genético para fins comercial e ambiental. O teste foi instalado em 1996 em Selvíria-MS, no delineamento experimental de blocos completos casualizados. Foram usados 60 tratamentos (progênies) sendo 30 da procedência de Ilha Solteira-SP e 30 de Selvíria-MS com cinco repetições, 10 plantas por parcela, no espaçamento de $3 \times 1,5 \mathrm{~m}$, em plantio alternado com Jacaranda cuspidifolia Mart. Foram mensurados os caracteres DAP (diâmetro a altura do peito), ALT (altura total da planta), DMC (diâmetro médio de copa), FOR (forma de fuste) aos 18 anos após o plantio e a identificação do sexo das árvores. As estimativas de componentes de variância e parâmetros genéticos foram obtidas via modelo misto REML/BLUP (máxima verossimilhança restrita/melhor predição linear não viciada). Observou-se diferenças significativas entre procedências para os caracteres DAP e ALT, na análise de cada procedência houve diferenças significativas entre e dentro de progênies para todos os caracteres, exceto FOR. O coeficiente de herdabilidade em nível de média de progênies variou de 0,43 (FOR-MS) a 0,78 (DAP-MS) e a herdabilidade individual de 0,08 (FOR-MS) a 0,37 (DAP-MS). A acurácia foi alta $(>0,65)$ e os coeficientes de variação genética foram maiores que 5,6 . As porcentagens de indivíduos em floração nas procedências de llha Solteira e Selvíria foram de $11 \%$ e $16 \%$, respectivamente, com predominância de indivíduos com flores masculinas. A estratégia de seleção dentro de progênies, com seleção de $50 \%$ dos indivíduos com base no caráter DAP, revelou baixos ganhos na seleção, porém é a mais indicada para esse teste, pois possivelmente levará a uma menor alteração da variabilidade genética da população e diminuirá a probabilidade de cruzamentos entre parentes. Essa proposta de desbaste seletivo é mais adequada visto que a porcentagem de indivíduos em florescimento é baixa, o que inviabiliza a identificação sexual de todos os indivíduos do teste, e, consequentemente, as estimativas mais acuradas de parâmetros genéticos como o tamanho efetivo populacional.
\end{abstract}

Palavras-chave: Conservação genética. Espécies nativas. Manejo florestal. Gonçalo alves.

\begin{abstract}
The objective was to propose a management plan for provenance and progeny tests of Astronium fraxinifolium Schott. It is based on knowledge of genetic variability in an effort to promote the conservation of their genetic potential for commercial and environmental purposes. The test was originally conducted in 1996 in Selvíria, Mato Grosso do Sul (MS) Brazil using an experimental of randomized complete block design. We applied a total of 30 treatments (progenies) of provenance in Ilha Solteira, São Paulo (SP) and 30 treatments of provenance in Selvíria, MS each having five repetitions, 10 plants per plot, spaced $3 \mathrm{~m} \times 1.5 \mathrm{~m}$ in alternate plantings with Jacaranda cuspidifolia. The traits DAP (height/diameter), ALT (total height), DMC (average crown diameter) and FOR (stem form) were measured 18 years after planting
\end{abstract}

\footnotetext{
${ }^{1}$ Mestre em Agronomia. UNESP - Universidade Estadual Paulista "Júlio de Mesquita Filho" / FEIS - Faculdade de Engenharia de Ilha Solteira. Av. Brasil, 56 - Caixa Postal 31 - 15378-000 - Ilha Solteira, SP, Brasil. E-mail: maiara cornacini@ hotmail.com; janainars t@hotmail.com; kellycristinadaluz@yahoo.com.br; wanderley.dossantos@hotmail.com.

2Doutor em Agronomia. UNESP - Universidade Estadual Paulista "Júlio de Mesquita Filho" / FEIS - Faculdade de Engenharia de Ilha Solteira. Av. Brasil-Centro, 56 - Caixa Postal 31 - 15378-000 - Ilha Solteira, SP, Brasil. E-mail: josecambuim@yahoo.com.br

3Professor Titular do Departamento de Fitotecnia e Tecnologia de Alimentos e Sócio Economia. UNESP - Universidade Estadual Paulista "Júlio de Mesquita Filho" / FEIS - Faculdade de Engenharia de Ilha Solteira. Av. Brasil-Centro, 56 - Caixa Postal 31 - 15378-000 - Ilha Solteira, SP, Brasil. E-mail: teixeira@agr.feis.unesp.br
}

${ }^{4}$ Pesquisadora Doutora. Embrapa Florestas. Estrada da Ribeira, km111 - Câmpus Samambaia - - Caixa-postal: 319 83411000 - Colombo, PR, Brasil. E-mail: ananda.aguiar@embrapa.br 
and sex of individuals. The estimates of variance components and genetic parameters were obtained by employing mixed models RELM/BLUP (Maximum restricted likelihood / best non-vitiated linear prediction). They detected significant differences among provenances for DAP and ALT. Analysis of each provenance indicated there were significant differences between and within progenies for all the traits, except FOR. The heritability coefficient of average level progenies ranged from 0.43 (FOR-MS) to 0.78 (DAP-MS) and individual heritability from 0.08 (FOR-MS) to 0.37 (DAP-MS). The accuracy was high $(>0.65)$ and the coefficient of genetic variations were higher than 5.6. From the total number $11 \%$ and $16 \%$ were flowering from SP and MS provenances, respectively; with a predominance of individuals with male flowers. The selection strategy within progenies, with selection of $50 \%$ of the individuals based on the DAP trait, revealed low gains. However, this test is more suitable because it may possibly lead to smaller alterations of the genetic variability of the population and reduce the likelihood of crossings between relatives. Therefore, this proposal for selective thinning is most appropriate because the flowering percentage of individuals is low, making it impractical for the determination of sex of total individuals. Consequently, the estimates of the parameters accurately affect size.

Keywords: Genetic conservation. Native species. Forest management. Gonçalo alves.

\section{INTRODUÇÃO}

O desmatamento e a fragmentação florestal são hoje grandes problemas ambientais no Brasil, pois promove perda da diversidade biológica. Na tentativa de conter e diminuir esses problemas, várias medidas foram tomadas nos últimos anos, tanto pelo poder público quanto pela pesquisa científica e tecnológica, impulsionadas pelo crescimento da preocupação com questões ambientais globais nas últimas décadas. Ainda assim, são alarmantes as taxas de desmatamento e extinção de espécies, uso ilegal de recursos biológicos e perda de conhecimentos tradicionais associados, sendo necessário que essas medidas sejam de fato executadas e que haja novas estratégias de práticas sustentáveis de uso dos recursos naturais (AMARAL et al., 1999; JAHNEL, 2008; GANEM, DRUMMOND, 2010; GANEM et al., 2013). Portanto, há uma demanda por conhecimento sobre espécies florestais nativas e também sementes com qualidade genética para o uso em reflorestamento com finalidade de recuperação ambiental e plantios comerciais. Apesar do Brasil ser um dos países de maior biodiversidade florestal, abrigando diversos biomas com grande número de espécies endêmicas, o uso dessas espécies é ainda muito restrito devido principalmente à falta de conhecimento de técnicas silviculturais e manejo, especificamente aquelas relacionadas à conservação e ao melhoramento genético.

Algumas áreas dos biomas brasileiros são tão exploradas que as espécies florestais que as compõem são encontradas, restritamente, à margem de rodovias ou em pequenas reservas ecológicas. Um exemplo é o Gonçalo alves (Astronium fraxinifolium Schott), árvore da família Anacardiaceae, que habita naturalmente a região de transição de Floresta Estacional Semidecidual e o Cerrado (LORENZI, 1998; AGUIAR, 2001). Atualmente, em condições naturais encontram-se poucos indivíduos dessa espécie (SANTOS et al., 2007). A excelente qualidade da sua madeira foi um dos motivos que contribuíram para sua exploração. Essa é dura, pesada e durável, muito usada na construção civil e naval e também utilizada na fabricação de mobiliário de luxo, balaústres, corrimões e portas nobres (LORENZI, 1998; MORAES NETO, 2008). Portanto, somente a conservação in situ não contribui para sua sobrevivência, em habitats totalmente degradados, desconectados. Além disso, de acordo com a Portaria IBAMA n. 37-N, de 3 de abril de 1992 (BRASIL, 1992), a espécie A. fraxinifolium está ameaçada de extinção fazendo-se necessário a conservação ex situ e a elaboração de estratégias que garantam a conservação da espécie e da sua variabilidade genética.

A conservação ex situ de um ou dois bancos de germoplasma em campo não garante a conservação da variabilidade genética das populações, pois esses podem ser facilmente perdidos por catástrofes naturais ou devido a fatores antropogênicos. Para uma conservação mais efetiva recomenda-se produzir sementes nesses bancos e utilizá-las em reflorestamentos para recuperação ambiental. Mas, para que esses bancos passem a produzir sementes com qualidade genética é necessário o seu manejo. Uma forma de manejo é o desbaste seletivo, o qual é aplicado no intuito de manter a taxa de crescimento e reduzir as chances de cruzamento entre árvores aparentadas, principalmente dentro das parcelas, quando estas são formadas por plantas da mesma progênie (SEBBENN et al., 
2009a; 2009b). O objetivo deste trabalho é estimar a variabilidade genética a partir de caracteres silviculturais e propor o manejo do teste de procedências e progênies de A. fraxinifolium, visando à conservação do seu potencial produtivo e genético para fins comercial e ambiental, principalmente quanto à produção de sementes com qualidade genética.

\section{MATERIAL E MÉTODOS}

As sementes usadas para implantação do teste de procedências e progênies de Astronium fraxinifolium Schott foram coletadas em 1995 de 30 árvores matrizes às margens da rodovia SP 595, entre os municípios de Ilha Solteira-SP e Santa Fé do Sul-SP, e 30 às margens da rodovia MS 158, no município de Selvíria-MS. O teste foi instalado em 26 de março de 1996 no delineamento experimental de blocos completos casualizados, com 60 tratamentos (famílias), 5 repetições e 10 plantas por parcela, no espaçamento de $3 \mathrm{~m} \times 1,5 \mathrm{~m}$, em plantio alternado com Jacaranda cuspidifolia. O experimento foi conduzido em condições de campo na Fazenda de Ensino e Pesquisa da FEIS/UNESP, situada à margem direita do rio Paraná, no município de Selvíria-MS, localizada nas coordenadas geográficas $20^{\circ} 20^{\prime} 08.64^{\prime \prime} \mathrm{S}, 5^{\circ} 24^{\prime} 16.04^{\prime \prime} \mathrm{W}$ e 378 metros de altitude. Aos 18 anos após o plantio foram avaliados a altura total de planta (ALT, em $\mathrm{m}$ ), o diâmetro a altura do peito (DAP, em $\mathrm{cm}$ ), diâmetro médio da copa (DMC, em m), forma do fuste (FOR, por atribuição de notas segundo Guerra, 2008) e sobrevivência ( $\mathrm{SOB}, \mathrm{em} \%)$. Por ser uma espécie dioica, também foi realizada a identificação sexual das árvores com base na visualização das flores masculinas e femininas.

As análises genético-estatísticas considerando o efeito de procedências foram obtidas pela metodologia do modelo linear misto (aditivo univariado) REML/BLUP, seguindo o procedimento proposto por Resende (2002a; 2007):

$$
y=\boldsymbol{X} r+\boldsymbol{Z} a+\boldsymbol{W} p+\boldsymbol{T} \boldsymbol{s}+e
$$

em que: y é o vetor de dados; $r$ é o vetor dos efeitos de repetição (assumidos como fixos) somados à média geral; $a$ é o vetor dos efeitos genéticos aditivos individuais (aleatórios); $p$ é o vetor dos efeitos de parcela (aleatórios); $s$ é vetor dos efeitos de população ou procedência (aleatórios), e é o vetor de erros ou resíduos (aleatórios). As letras maiúsculas representam as matrizes de incidência para os referidos efeitos.

Nas análises individuais, ignorando o efeito de procedências, foi considerado o seguinte modelo estatístico:

$$
y=\boldsymbol{X} r+\boldsymbol{Z} a+\boldsymbol{W} p+e ;
$$

em que: $y$ é o vetor de dados; $r$ é o vetor dos efeitos de repetição (assumidos como fixos) somados à média geral; $a$ é o vetor dos efeitos genéticos aditivos individuais (aleatórios); $p$ é o vetor dos efeitos de parcelas (aleatórios), e é o vetor de erros ou resíduos (aleatórios). As letras maiúsculas representam as matrizes de incidência para os referidos efeitos.

Foram estimados os seguintes parâmetros: Herdabilidade individual no sentido restrito $\left(\hat{h}_{a}^{2}\right)$, Herdabilidade em nível de média de progênies $\left(\hat{h}_{m}^{2}\right)$, Herdabilidade aditiva dentro de parcela $\left(\hat{h}_{a d}^{2}\right)$, Coeficiente de variação genética aditiva individual $\left(C V_{g i}\right)$, Coeficiente de variação genotípica entre progênies $\left(C V_{g p}\right)$, Coeficiente de variação experimental $\left(C V_{e}\right)$, Coeficiente de variação relativa $\left(C V_{r}\right)$, Acurácia da seleção de progênies $\left(r_{a \alpha}\right)$, Coeficiente de determinação dos efeitos de parcela $\left(\hat{C}_{p}^{2}\right)$.

Com base nos maiores valores genéticos preditos individuais (BLUP) foram propostas duas estratégias de seleção, tendo por base o DAP, considerando tanto a conservação quanto o melhoramento genético:

a) Seleção dos 750 melhores indivíduos com base nos maiores valores genéticos individuais (BLUP) em cada procedência. As progênies selecionadas poderiam ter qualquer número de indivíduos entre 1 a 50.

b) Seleção de 50\% dentro das progênies, o que corresponde a um total de 750 indivíduos selecionados: 30 (progênies) x 5 (plantas por progênie) x 5 (repetições) para as duas procedências. Nessa situação, todas as progênies tiveram um total de 25 indivíduos selecionados.

O ganho genético em porcentagem (Gs\%) com a seleção corresponde a: 


$$
G s(\%)=\left(\frac{\sum \hat{a}}{\sum k /} \times 100\right) \div \hat{m}
$$

em que $\hat{a}$ corresponde aos valores genéticos preditos individuais (BLUP); $k f$ é o número médio de indivíduos selecionados por progênie e ${ }^{m}$ é a média geral do caráter.

O tamanho efetivo populacional $\left(N_{e}\right)$ para A. fraxinifolium foi estimado com base no método descrito por Vencovsky et al. (2012) para espécies dioicas. Esse parâmetro foi estimado antes e após a aplicação das propostas de seleção descritas acima. Para isso, assumiu-se que a população de origem era de tamanho arbitrário com exclusão parcial dos pais e que houve controle gamético apenas do lado feminino, pois foram coletados o mesmo número de sementes (50) de cada árvore matriz feminina. Assim, o tamanho efetivo foi estimado conforme a expressão:

$$
N_{e} \approx 4 t / D_{7}
$$

em que, $t=f+m$, sendo $f$ número de indivíduos fêmeas e $m$ número de indivíduos machos no experimento (filhos), respectivamente, $\mathrm{e}$

$$
D_{7}=\frac{1}{r(1-r)}+t \frac{(1-u) t}{F}+\frac{(1-v) t-1}{M}-1
$$

sendo $u=F / N f$ e $v=M / N m, N f=$ número de fêmeas e $N m$ = número de machos da população de origem, $F$ = fêmeas que participaram da reprodução e $M=$ machos que participaram da reprodução, $r=$ proporção sexual dos filhos, dada por: $r=f /(f+m)$.

A diversidade genética $(D)$, após a seleção, foi quantificada conforme Wei e Lindgren (1996), citados por Resende (2002b):

$$
D=N_{e f} / N_{f o}
$$

Em que: $0<D \leq 1$, sendo: $N_{f o}=$ número original de famílias, $N_{e f}=$ número efetivo de famílias selecionadas, sendo dado por: $N_{e f}=\left(\sum k_{f}\right)^{2} / \sum k_{f}^{2}$.

\section{RESULTADOS E DISCUSSÃO}

\section{Variabilidade genética}

$\mathrm{Na}$ análise da deviance, considerando as duas procedências, diferenças significativas foram observadas entre as procedências e entre e dentro de progênies de A. fraxinilifolium para os caracteres altura (ALT), diâmetro a altura do peito (DAP), diâmetro médio de copa (DMC) e sobrevivência (SOB), com exceção do DMC entre procedências e SOB dentro de progênies (Tabela 1). Diferenças significativas entre as procedências não foram observadas por Aguiar et al. (2003) aos quatro anos de idade para os caracteres ALT, DMC e forma (FOR), neste mesmo experimento. Apesar das populações naturais encontrarem-se em ambientes antropizados, a variação genética existente entre e dentro de procedências foi conservada. Em geral, as espécies nativas têm crescimento lento e os coeficientes estimados para alguns caracteres podem vir a mudar e expressar variação genética em idades mais avançadas, quando atingirem a maturidade reprodutiva (SEBBENN et al., 1998). Além disso, esse resultado pode indicar a ocorrência de alterações no comportamento das procedências e progênies para os caracteres estudados (SEBBENN et al., 2009b). Siqueira et al. (1993) acreditam que estimativas dos parâmetros ao longo dos anos são necessárias, principalmente até a meia idade de corte, e podem permitir que se conheça melhor a estrutura genética das populações estudadas.

Diferenças entre procedências para caracteres de crescimento foram observadas em outras espécies, tais como: Balfoudendron riedelianum, Cariniana legalis e Gallesia integrifolia (KUBOTA et al., 2015; SEBBENN et al., 2007; 2009a; 2009b). Batista et al. (2012) estudando três procedências de Handroanthus vellosoi verificaram que o efeito de procedência foi significativo apenas para forma do fuste, o que não foi o caso desse teste, em que a FOR não apresentou diferenças significativas entre procedências, progênies e parcelas. Quando se trata de seleção para fins de melhoramento deve-se priorizar indivíduos das procedências mais produtivas e com maior valor genético aditivo. Para conservação da variabilidade genética indivíduos mais adaptados (alta sobrevivência, desempenho e com boa produção de flores) das duas procedências deverão ser mantidos no ensaio para evitar perdas de variabilidade genética e, consequentemente de alelos raros. 
As médias gerais, considerando as duas procedências, foram de 11,12 cm, 9,08 m, 4,12 m, 3,71 e 76\% para DAP, ALT, DMC, FOR e SOB, respectivamente (Tabela 1). Apesar das progênies de Ilha Solteira terem-se adaptado bem ao local de plantio, a procedência de Selvíria apresentou médias superiores a de Ilha Solteira para todos os caracteres de crescimento (Tabela 2). Isso, provavelmente deve-se ao seu potencial genético produtivo ou às características pedológicas do local de plantio, tendo em vista que as condições climáticas nos dois locais de ocorrência natural e do plantio são muito semelhantes.

Tabela 1. Análise de deviance para os caracteres silviculturais em um teste de procedências e progênies de Astronium fraxinifolium, aos 18 anos em plantio consorciado com progênies de Jacaranda cuspidifolia, em Selvíria-MS.

Table 1. Deviance analysis for silvicultural traits in a provenance and progenies test of Astronium fraxinifolium, to 18 years old in intercropping with progenies of Jacaranda cuspidifolia in Selvíria-MS.

\begin{tabular}{lcccc}
\hline \multirow{2}{*}{ Caracteres } & $\hat{m}$ & \multicolumn{1}{c}{ LRT } & Parcelas \\
\cline { 3 - 5 } & & Procedências & Progênies & $5,61^{*}$ \\
DAP & $11,12 \mathrm{~cm}$ & $9,18^{* *}$ & $23,8^{\star *}$ & $20,95^{* *}$ \\
DLT & $9,08 \mathrm{~m}$ & $37,04^{* *}$ & $10,58^{* *}$ & $9,22^{* *}$ \\
FOR & $4,12 \mathrm{~m}$ & $0,001^{\text {ns }}$ & $12,3^{\star *}$ & $2,39^{\text {ns }}$ \\
SOB & 3,71 & $0,12^{\text {ns }}$ & $1,53^{\text {ns }}$ & $7,67^{* *}$ \\
\hline
\end{tabular}

DAP: diamêtro a altura do peito, ALT: altura, DMC: diamêtro médio da copa,

FOR: forma de fuste, SOB: sobrevivência,

LRT: Teste da razão de verossimilhança, $\hat{m}$ : média, *** significativo a $1 \%$, * significativo a $5 \%$. ${ }^{\text {ns }}$ não significativo.

Atualmente, o teste encontra-se com 1.116 e 1.180 indivíduos das procedências de Ilha Solteira e Selvíria, respectivamente. As progênies de A. fraxinifolium aos 18 anos tiveram taxa de sobrevivência de $74 \%$ para procedência de Ilha Solteira e 78\% para a de Selvíria, indicando boa adaptação da espécie ao local de plantio, principalmente se compararmos a média de sobrevivência de espécies nativas (Tabela 2). Aos quatro anos de idade a taxa de sobrevivência do teste era de $81 \%$ (AGUIAR et al., 2003). Durante os primeiros anos após o plantio houve uma mortalidade maior se comparada aos anos seguintes, umas das hipóteses é a não adaptação de alguns indivíduos às condições pedoclimáticas. Segundo Sebbenn et al. (2009b) a taxa de mortalidade é maior em idade precoce devido à seleção natural contra indivíduos endogâmicos.

$\mathrm{Na}$ análise individual de cada procedência (Tabela 2), existem diferenças significativas para o efeito de progênies e parcelas para os caracteres avaliados, exceto para FOR na procedência de Selvíria. Este resultado permite inferir que o delineamento experimental utilizado foi adequado para controlar o ambiente e efetivo para detectar variação entre e dentro de progênies. Souza et al. (2003) não observaram diferenças significativas para todos os caracteres em progênies de A. fraxinilifolium procedentes de Selvíria-MS aos quatro anos de idade. Segundo os autores, provavelmente não houve tempo para a expressão da variação entre as progênies por se encontrar muito juvenil, o que justifica a necessidade de estudos mais prolongados para a espécie.

A procedência de Selvíria apresentou valores médios superiores à de Ilha Solteira para todos os caracteres avaliados; 9,54 m e 8,62 m (ALT); 11,45 cm e 10,78 cm (DAP); 4,14 m e 4,10 m (DMC) e 3,73 e 3,69 (FOR) (Tabela 2). Aguiar et al. (2003) também observaram resultados semelhantes nos quatros primeiros anos após o plantio para esse mesmo experimento. Otsubo et al. (2015) também observaram que uma procedência $A$. fraxinilifolium de Selvíria-MS apresentou desempenho em crescimento superior aos 14 anos de idade $(8,60 \mathrm{~m}, 9,40 \mathrm{~cm} \mathrm{e} \mathrm{3,10} \mathrm{m;} \mathrm{para} \mathrm{ALT,} \mathrm{DAP} \mathrm{e} \mathrm{DMC,}$ respectivamente) se comparada à média deste teste, porém a FOR foi inferior $(3,42)$. O efeito de competição ocorrido entre os indivíduos da mesma espécie e de J. cuspidifolia pode ter contribuído para o menor desempenho dessa procedência. Por outro lado, o plantio consorciado com o J. cuspidifolia favoreceu uma boa forma para o A. fraxinifolium. Souza et al. (2003) verificaram que o sistema de plantio consorciado foi menos favorável para o desenvolvimento das plantas de A. fraxinilifolium, porém proporcionou uma melhor forma de fuste. Portanto, o plantio dessa espécie em sistema heterogêneo e menos adensado deve ser considerado, principalmente, se a finalidade for madeira para desdobro. 


\section{Estimativas de parâmetros genéticos}

O controle da variação experimental, bem como dentro de parcelas, foi eficiente no teste, o que pode ser confirmado a partir dos coeficientes de variação experimental, variação relativa e de parcela (Tabela 2). De maneira geral, o $C V_{e}$ para procedência de Ilha Solteira foi maior que de Selvíria para todos os caracteres. Valores desse coeficiente na ordem de 10 a $20 \%$ podem ser considerados baixos para experimentos em que ocorre competição entre plantas (PIMENTEL-GOMES; GARCIA, 2002). O efeito da heterogeneidade ambiental dentro dos blocos não foi verificado para todos os caracteres, conforme as estimativas do coeficiente de determinação dos efeitos de parcelas ( $\left.C_{\text {parr. }}^{2}\right)$, que variaram de e $1 \%$ a $11 \%$. A procedência de Selvíria apresentou maior coeficiente de variação relativa $\left(C V_{r}\right.$ ) para DAP e FOR e a de Ilha Solteira para o DAP e ALT. Para sucesso na seleção o caráter DAP é o mais indicado pelo fato de ter maiores valores de $C V_{r}$ para as duas procedências, além de ser de fácil mensuração e tem alta correlação com a altura.

As estimativas do coeficiente de herdabilidade individual no sentido restrito variaram de 0,08 a 0,37. Os valores desse coeficiente são considerados altos para o DAP e a ALT da procedência de Selvíria, moderados para os caracteres DAP, ALT, DMC e FOR para a de Ilha Solteira e para o DMC de Selvíria. Esse parâmetro genético é fundamental na predição de ganho devido à seleção, expressando-se a confiança do valor fenotípico como estimador do valor genético (FALCONER; MACKAY, 1996). As estimativas de herdabilidade com base em média de progênies ( $\hat{h}_{m}^{2}$ ) foram altas para todos os caracteres nas duas procedências $(0,59$ a 0,78$)$, exceto para FOR na procedência de Selvíria $(0,43)$. A herdabilidade aditiva dentro de parcela $\left(\hat{h}_{a d}^{2}\right)$ foi semelhante a $\hat{h}_{a}^{2}$ variando de 0,07 a 0,32 (Tabela 2). Otsubo et al. (2015), estudando uma população de A. fraxinilifolium aos 15 anos de idade, verificaram herdabilidades moderadas para os caracteres ALT, DAP, DMC e FOR. Segundo os autores, os valores variaram de 0,17 a 0,22 para $\hat{h}_{a}^{2} ; 0,51$ a 0,57 para $\hat{h}_{m}^{2}$ e 0,14 a 0,19 para $\hat{h}_{\text {ad }}^{2}$.

O caráter DAP foi o que teve os maiores valores de herdabilidades na procedência de Selvíria. Na procedência de Ilha Solteira o DAP teve estimativas maiores somente para o coeficiente de herdabilidade em nível de média de progênies. Altas herdabilidades indicam um bom controle genético o que possibilita progressos genéticos com a seleção entre progênies. Portanto, a população possui potencial evolutivo para responder às mudanças ambientais, já que parte significativa da variação fenotípica observada nos caracteres é de origem genética (FREITAS et al., 2006; SEBBENN et al., 2009b).

As estimativas de herdabilidades ( $h_{m}^{2}$ e $\left.h_{a d}^{2}\right)$, acurácia $\left(r_{a a}\right)$ e do coeficiente de variação relativa $\left(C V_{r}\right)$ foram altas para os caracteres ALT, DAP e DMC. Sugere-se o uso desses caracteres no processo de seleção individual ou entre progênies. Portanto, as procedências têm uma base genética ampla e se for explorada de maneira adequada, poderá gerar ganhos significativos ao longo das gerações de seleção. A seleção das melhores progênies ou árvores de cada progênie proporcionará a formação de um Pomar de Sementes por Mudas que pode ser utilizado para diversos fins.

A procedência de Selvíria teve um coeficiente de variação genético $\left(C V_{g i}\right.$ e $\left.C V_{g p}\right)$ maior do que a procedência de Ilha Solteira para DAP e ALT. Com base nos resultados obtidos para algumas espécies nativas, tais como H. vellosoi, B. riedelianum e Myracrodruon urundeuva (BATISTA et al., 2012; FREITAS et al., 2007; SEBBENN et al., 2007), pode-se concluir que para as duas populações em estudo foram encontrados valores moderados a altos variando de $6,11 \%$ a $24,33 \%$. As acurácias foram altas para todos os caracteres de crescimento estudados $(0,76$ a 0,89$)$, exceto paro FOR da procedência de Selvíria $(0,65)$, que mesmo assim, é considerado adequado segundo Resende (2002a).

\section{Seleção de indivíduos}

Nesta estação reprodutiva de um total de 1116 indivíduos na procedência de Ilha Solteira foram observadas flores em 125 e de 1180 na de Selvíria em 191 árvores. A procedência de Selvíria teve uma porcentagem maior de florescimento (16\%) que a de Ilha Solteira (11\%). Do total de árvores que floresceram, $72 \%$ e $70 \%$ são árvores masculinas e $28 \%$ e $30 \%$ femininas, respectivamente para Selvíria e Ilha Solteira.

A taxa de florescimento do A. fraxinifolium foi baixa se comparada à espécie M. urundeuva (BERTONHA et al., 2016). Esses autores verificaram florescimento de 80,9\% para M. urundeuva em sistema de plantio homogêneo e 41,8\% em sistema misto com Corymbia citriodora aos 16 anos. Assim como no presente trabalho, os autores constataram uma predominância de florescimento de plantas masculinas, aproximadamente 5:1 (BERTONHA et al., 2016). Esses resultados diferem da razão sexu- 
al encontrada na natureza, 1:1, como sugere Opler e Bawa (1978) e também em estudos para as espécies Virola sebifera (LENZA; OLIVEIRA, 2006), Tapirira guianensis (LENZA; OLIVEIRA, 2005), Araucaria angustifolia (PALUDO et al., 2009) e Neea theifera (AMORIM et al., 2011). Contudo, Amorim e Oliveira (2006) verificaram para Amaioua guianensis razão sexual de 1,49 para o sexo masculino. No presente teste, o ideal seria realizar a identificação sexual de todos os indivíduos antes de realizar um desbaste seletivo. Porém, acredita-se que o desbaste seletivo de menor intensidade, eliminando somente os indivíduos inferiores, será necessário para proporcionar espaço e luz suficiente para desenvolvimento da copa, e consequentemente, para que os indivíduos selecionados possam florescer. Após o florescimento dos indivíduos é necessária a identificação sexual para que em futuras seleções predomine a razão sexual de 1:1, com a proposta de manter maior variabilidade genética.

Tabela 2. Estimativa de parâmetros genéticos para diâmetro a altura do peito (DAP), altura total (ALT), diâmetro médio da copa (DMC) e forma de fuste (FOR) aos 18 anos de idade de um do teste de progênies e procedência de Astronium fraxinifolium em plantio consorciado com progênies Jacaranda cuspidifolia Mart, em Selvíria-MS.

Table 2. Estimates of genetic parameters for breast height diameter (DAP), total height (ALT), average crown diameter (DMC) and stem form (FOR) at 18 years old in provenance and progenies test of Astronium fraxinifolium in intercropping with progenies Jacaranda cuspidifolia, in Selvíria-MS.

\begin{tabular}{|c|c|c|c|c|c|c|c|c|c|c|c|c|c|}
\hline Procedência & Caracteres & $\hat{m}$ & $\hat{h}_{a}^{2}$ & $C_{\text {parc! }}^{2}$ & $\hat{i}_{m p}^{2}$ & $r_{\hat{a} a}$ & $\hat{h}_{a d}^{2}$ & $C V_{g i}$ & $C V_{g p}$ & $C V_{e}$ & $C V_{r}$ & $\begin{array}{l}\text { LRT }\left(X^{2}\right) \\
\text { prog }\end{array}$ & $\begin{array}{c}\text { LRT }\left(X^{2}\right) \\
\text { parc }\end{array}$ \\
\hline \multirow{4}{*}{ Ilha Solteira } & $\mathrm{DAP}(\mathrm{cm})$ & 10,78 & $\begin{array}{c}0,28 \\
( \pm 0,09)\end{array}$ & 0,05 & 0,71 & 0,84 & 0,23 & 21,85 & 10,42 & 15,59 & 0,70 & $16,7^{* *}$ & $8,22^{* *}$ \\
\hline & $\operatorname{ALT}(\mathrm{m})$ & 8,62 & $\begin{array}{c}0,27 \\
( \pm 0,09)\end{array}$ & 0,10 & 0,64 & 0,80 & 0,24 & 18,86 & 9,43 & 15,64 & 0,60 & $23,89^{\star *}$ & $23,89^{* *}$ \\
\hline & $\mathrm{DMC}(\mathrm{m})$ & 4,10 & $\begin{array}{c}0,29 \\
( \pm 0,9)\end{array}$ & 0,06 & 0,69 & 0,83 & 0,23 & 15,66 & 8,25 & 12,32 & 0,67 & $14,58^{* *}$ & $10,45^{\star \star}$ \\
\hline & FOR & 3,69 & $\begin{array}{c}0,28 \\
( \pm 0,09)\end{array}$ & 0,03 & 0,74 & 0,86 & 0,23 & 11,36 & 5,68 & 7,45 & 0,76 & $18,52^{\star *}$ & $3,18^{\text {ns }}$ \\
\hline \multirow{4}{*}{ Selvíria } & $\mathrm{DAP}(\mathrm{cm})$ & 11,45 & $\begin{array}{c}0,37 \\
( \pm 0,10)\end{array}$ & 0,04 & 0,78 & 0,88 & 0,32 & 24,33 & 12,16 & 14,34 & 0,84 & $26,82^{* *}$ & $6,82^{* *}$ \\
\hline & $\operatorname{ALT}(\mathrm{m})$ & 9,54 & $\begin{array}{c}0,35 \\
( \pm 0,1)\end{array}$ & 0,07 & 0,73 & 0,85 & 0,31 & 19,82 & 9,91 & 13,46 & 0,73 & $19,62^{\star *}$ & $16,62^{\star *}$ \\
\hline & $\mathrm{DMC}(\mathrm{m})$ & 4,14 & $\begin{array}{c}0,22 \\
( \pm 0,08)\end{array}$ & 0,11 & 0,59 & 0,76 & 0,20 & 13,60 & 6,8 & 12,76 & 0,53 & $8,82^{* *}$ & $31,2^{* *}$ \\
\hline & FOR & 3,73 & $\begin{array}{c}0,08 \\
( \pm 0,04)\end{array}$ & 0,05 & 0,43 & 0,65 & 0,07 & 5,66 & 2,83 & 7,22 & 0,39 & $2,98^{\text {ns }}$ & $7,22^{* *}$ \\
\hline
\end{tabular}

Entender a relação entre o tamanho efetivo populacional $\left(N_{e}\right)$ e o tamanho real de uma população é de fundamental importância para planejar estratégias de manejo para a conservação. $\mathrm{O} N_{e}$ foi calculado considerando cruzamentos perfeitamente aleatórios e com a possibilidade de as progênies amostradas terem recebido pólen de diferentes conjuntos gênicos. Também foi considerada a possibilidade de florescimento de todos os indivíduos em proporção de 1:1 de machos e fêmeas. Para procedência de Ilha Solteira observou-se um $N_{e}$ de 108,35 e para a de Selvíria de 108,92, sem considerar a seleção. Segundo Vencovsky (1987) um $N_{e}$ da ordem de 50 em conservação ex situ consegue reter alelos com frequência $\geq 6 \%$, porém para reter alelos com frequência $\geq 1 \%$ é necessário um $N_{e}$ da ordem de 150. Para atingir esse valor devem ser amostradas pelo menos 100 sementes de cerca de 40 matrizes da natureza, em populações alógamas.

$\mathrm{O} N_{e}$ nesse experimento poderia aumentar se considerar as duas procedências como uma única população. Porém, isso não foi considerado porque houve diferença significativa entre elas. Cruzamentos entre os indivíduos das duas procedências podem ocorrer naturalmente pelo fato dos indivíduos de cada procedência terem sido plantados intercaladamente, assim não encontram barreiras geográficas. No futuro, estudos com aplicação de ferramentas moleculares poderão ser úteis para esclarecer a estrutura genética e o grau de parentesco entre os indivíduos desse teste, e dos que serão estabelecidos com as sementes procedentes. Portanto, para uma conservação mais efetiva propõe-se 
aplicar uma seleção de menor intensidade no teste, em torno de 50\% dos indivíduos. Do total de indivíduos do teste, foram selecionados os 750 melhores indivíduos remanescentes de cada procedência, considerando duas formas de seleção: seleção dos melhores indivíduos sendo $k f=25$ para todas as progênies $(k f=k \neq 0)$ e para outra seleção os melhores indivíduos independentemente do número de indivíduos por progênie.

Após a seleção, o tamanho efetivo populacional para as duas procedências e para os dois tipos de seleção foi o mesmo $(103,43)$. Isso ocorreu porque na fórmula de tamanho efetivo populacional (VENCOVSKY et al., 2012) foi considerado o mesmo número de indivíduos (750 indivíduos), intensidade de seleção (50\%) e razão sexual para as duas procedências. A seleção aplicada afetou pouco o tamanho efetivo populacional do teste (Tabela 3). Após o florescimento de uma porcentagem maior de indivíduos (acima de 50\%) sugere-se estimar novamente o tamanho efetivo populacional antes de uma nova proposta de manejo seletivo. Assim, a real razão sexual e/ou outros valores poderão ser considerados.

A diversidade $(D)$ do experimento após a seleção da procedência de Ilha Solteira e Selvíria correspondem a aproximadamente 1,00 considerando o $k f$ fixo. Para o $k f$ variável, $D$ foi de 0,82 e 0,81 para Ilha Solteira e Selvíria, respectivamente (Tabela 3). Indicando que a seleção com o $k f$ fixo mantém a diversidade genética inicial, enquanto que a seleção com o $k f$ variável houve uma diminuição dessa diversidade $(D)$. Esses resultados são compatíveis a outros trabalhos com espécies nativas (FREITAS et al., 2006; 2008; KUBOTA et al., 2015; SEBBENN et al., 2007; 2009a,b; GUERRA et al., 2009).

O ganho com a seleção foi superior para a procedência de Selvíria nas duas formas de seleção ( $k f$ fixo: 6,12\% e $k f$ variável: 9,13\%) quando comparado com a procedência de Ilha Solteira ( $k f$ fixo: 3,68\% e $k f$ variável: 6,45\%) (Tabela 3). Porém, as duas procedências tiveram ganhos inferiores em relação a outra população desta espécie estabelecida em outro ensaio (OTSUBO et al., 2015). Para todas as populações o ganho com seleção foi maior com a estratégia de $k f$ variável.

Considerando baixa frequência desta espécie em remanescentes de florestas estacionais decíduas no domínio do cerrado, quando comparada com outras espécies (SANTOS et al., 2007), e pelo fato da espécie estar ameaçada de extinção, faz-se necessária a sua conservação. Portanto, como estratégia de seleção será adequada a utilização do $k f$ fixo, que apesar de resultar ganho de seleção baixo, permitirá que a variabilidade genética da população seja mantida. Além disso, espera-se que diminua a probabilidade de cruzamentos entre parentes para o fornecimento de sementes com menor endogamia e potencial evolutivo para reflorestamentos ambientais.

Tabela 3. Ganho na seleção baseado no DAP em progênies de Astronium fraxinifolium procedentes da região de Ilha Solteira-SP e Selvíria-MS e consorciado com progênies Jacaranda cuspidifolia, em duas condições: $\mathrm{kf}=\mathrm{k}$ $\neq 0, \mathrm{kf}=\forall \mathrm{k} \neq 0$, aos 18 anos de idade em Selvíria-MS.

Table 3. Selection gain based on DAP in progenies of Astronium fraxinifolium from the region of Ilha Solteira-SP and Selvíria-MS, intercropped with progenies of Jacaranda cuspidifolia in two conditions: $\mathrm{kf}=\mathrm{k} \neq 0, \mathrm{kf}=$ $\forall \mathrm{k} \neq 0$, at 18 years old in Selvíria- MS.

\begin{tabular}{llllllllllll}
\hline \multirow{2}{*}{ Procedência } & Estratégia & $n$ & $N_{f}$ & $N_{f}$ & $\bar{k}_{f}$ & $\hat{\sigma}_{h f}^{2}$ & $N_{e}$ & $\mu(\mathrm{cm})$ & $\hat{a}(\mathrm{~cm})$ & $\hat{G}_{s}(\%)$ & $\hat{D}$ \\
\hline \multirow{2}{*}{ Ilha Solteira } & $\mathrm{kf}=\mathrm{k} \neq 0$ & 749 & 30 & 30 & 24,97 & 0,033 & 103,43 & 12,47 & 0,40 & 3,68 & 0,99 \\
& $\mathrm{kf}=\forall \mathrm{k} \neq 0$ & 750 & 30 & 30 & 25 & 140,27 & 103,45 & 12,51 & 0,69 & 6,45 & 0,82 \\
\multirow{2}{*}{ Selvíria } & $\mathrm{kf}=\mathrm{k} \neq 0$ & 750 & 30 & 30 & 25 & 0 & 103,45 & 13,64 & 0,70 & 6,12 & 1,00 \\
& $\mathrm{kf}=\forall \quad \mathrm{k} \neq 0$ & 750 & 30 & 30 & 25 & 149,31 & 103,45 & 13,61 & 1,045 & 9,13 & 0,81 \\
\hline
\end{tabular}

Número de indivíduos selecionados $\left({ }^{n}\right)$; número original de famílias $\left(N_{6}\right)$; número de famílias selecionadas $\left(N_{f}\right)$; número médio de indivíduos selecionados por família $\left(\bar{k}_{f}\right)$; variância do número de indivíduos selecionados por família ( $\left.\hat{\sigma}_{h f}^{2}\right)$; tamanho efetivo populacional ( $N_{\epsilon}$ ); média do experimento para o DAP ( $\mu$ ); índice multi-efeitos ( $\hat{a})$; ganho de seleção $\left(G_{s}\right)$; diversidade genética $(\hat{D})$.

\section{CONCLUSÕES}

As procedências de Astronium fraxinilifolium, de Ilha Solteira (SP) e Selvíria (MS), diferem entre si para os caracteres DAP e ALT aos 18 anos após o plantio. Existem diferenças significativas entre e dentro de progênies para os caracteres avaliados. As progênies de Selvíria tem desempenho em crescimento superior quando comparado às de Ilha Solteira. 
Para uma efetiva conservação genética dessa espécie e formação de um pomar de sementes por mudas a estratégia de seleção de 50\% de mesmo número de indivíduos dentro de progênies será mais efetiva para manter a variabilidade genética da população e diminuir a probabilidade de cruzamentos entre parentes.

\section{REFERÊNCIAS BIBLIOGRÁFICAS}

AGUIAR, A. V. Variação genética em progênies de Astronium fraxinolium Schott e Jacaranda cuspidifolia Mart. em consorcio. 2001. 126 p. Dissertação (Mestrado em Agronomia) - Faculdade de Engenharia, Universidade Estadual Paulista, Ilha Solteira, 2001.

AGUIAR, A. V.; BORTOLOZO, F. R.; MORAES, M. L. T.; ANDRADE, J. A. C. Genetic variation in Astronium fraxinifolium populations in consortium. Crop Breeding and Applied Biotechonology, Viçosa, v. 3, n. 2, p. 95-106, 2003.

AMARAL, W. A. N.; BRITO, M. C. W.; ASSAD, A. L. D.; MANFIO, G. P. Políticas públicas em biodiversidade: conservação e uso sustentado no país da megadiversidade. International Studies on Law and Education, v.1, p. 1-10, 1999

AMORIM, F. W.; MENDES-RODRIGUES, C.; MARUYAMA, P. K.; OLIVEIRA, P. E. Sexual ratio and floral biology of the dioecious Neea theifera Oerst. (Nyctaginaceae) in a cerrado rupestre of central Brazil. Acta Botânica Brasilica, Porto Alegre, v. 25, n. 4, p. 785-792, 2011.

AMORIM, F. W.; OLIVEIRA, P. E. Estrutura sexual e ecologia reprodutiva de Amaioua guianensis Aubl. (Rubiaceae), uma espécie dióica de formações florestais de cerrado. Revista Brasileira de Botânica, São Paulo, v. 29, n. 3, p. 353-362, 2006.

BATISTA, C. M.; FREITAS, M. L. M.; MORAES, M. A.; ZANATTO, A. C. S.; SANTOS, P. C.; ZANATA, M.; MORAES, M. L. T.; SEBBENN, A. M. Estimativas de parâmetros genéticos e a variabilidade entre e entro de procedências de Handroanthus vellosoi. Pesquisa Florestal Brasileira, Colombo, v. 32, n. 71, p. 269 - 276, 2012.

BERTONHA, L. J.; FREITAS, M. L. M.; CAMBUIM, J.; MORAES, M. L. T.; SEBBENN, A. M. Seleção de progênies de Myracrodruon urundeuva baseada em caracteres fenológicos e de crescimento para reconstituição de áreas de Reserva Legal. Scientia Forestalis, Piracicaba, v. 44, n. 109 p. 95104, 2016.

FALCONER, D. S.; MACKAY, T. F. C. Introduction to quantitative genetics. 4. ed. New York: Longman, 1996. $464 \mathrm{p}$.

FREITAS, M. L. M.; SEBBENN, A. M.; ZANATTO, A. C. S.; MORAES, E.; MORAES, M. A. Variação genética para caracteres quantitativos em população de Gallesia integrifolia (Spreng.) Harms. Revista do Instituto Florestal, São Paulo, v. 20, n. 2, p. 165-173, 2008.

FREITAS, M. L. M.; SEBBENN, A. M.; ZANATTO, A. C. S.; MORAIS, E. Formação de pomar de sementes a partir da seleção dentro de teste progênies de Myracrodruon urundeuva. Revista do Instituto Florestal, São Paulo, v. 19, n. 2, p. 65-72, 2007.

FREITAS, M. L. M.; SEBBENN, A. M.; MORAIS, E.; ZANATTO, A. C. S.; VERARDI, C. K.; PINHEIRO, A. N. Parâmetros genéticos em progênies de polinização aberta de Cordia trichotoma (Vell.) ex Steud. Revista do Instituto Florestal, São Paulo, v. 18, p. 95-102, 2006.

GANEM, R. S.; DRUMMOND, J. A. Biologia da conservação: as bases científicas da proteção da biodiversidade. in: GANEM, R.S. (Org.). Conservação da biodiversidade: legislação e políticas públicas. Brasília: Câmara dos Deputados, 2010. p. 11-46. 
Cornacini et al. - Desbaste seletivo em teste de procedências e progênies

de Astronium fraxinifolium Schott com base na variabilidade genética

GANEM, R. S.; DRUMMOND, J. A.; FRANCO, J. L. A. Conservation policies and control of habitat fragmentation in the Brazilian Cerrado biome. Ambiente \& Sociedade, Campinas, v. 16, p. 99-118, 2013.

GUERRA, C. R. S. B. Conservação genética Ex Situ de populações naturais de Myracrodruon urundeuva Fr.All. em sistema silvipastoril. 2008. 108 p. Tese (Doutorado em Sistema de Produção) - Faculdade de Engenharia, Universidade Estadual Paulista, Ilha Solteira, 2008.

GUERRA, C. R. S. B.; MORAES, M. L. T.; SILVA, C. L. S. P.; CANUTO, D. S. O.; ANDRADE, J. A. C.; FREITAS, M. L. M.; SEBBENN, A. M. Estratégias de seleção dentro de progênies em duas populações de Myracrodruon urundeuva Fr. All. Scientia Forestalis, Piracicaba, v. 37, n. 81, p. 79-87, 2009.

BRASIL. IBAMA - INSTITUTO BRASILEIRO DO MEIO AMBIENTE E DOS RECURSOS NATURAIS. Portaria No 37-N, 3 de abril de 1992. Disponível em: < http://www.cetesb.sp.gov.br/licenciamentoo/legislacao/ federal/portarias/1992 Port IBAMA 37.pdf > Acesso em: 10 jan. 2014.

JAHNEL, V. Proposta para delineamento de um pomar de sementes de espécies florestais nativas. 2008. 50 p. Monografia (Graduação em Engenharia Florestal) - Universidade Federal Rural do Rio de Janeiro, Seropédica, 2008.

KUBOTA, T. Y. K.; MORAES, M. A.; SILVA, E. C. B.; PUPIN, S.; AGUIAR, A. V.; MORAES, M. L. T.; FREITAS, M. L. M.; SATO, A. S.; MACHADO, J. A. R.; SEBBENN, A.M. Variabilidade genética para caracteres siilviculturais em progênies de polinização aberta de Balfourodendron riedelianum (Engler). Scientia Forestalis, Piracicaba, v. 43, n. 106, p. 407-415, 2015.

LENZA, E.; OLIVEIRA, P. E. Biologia reprodutiva e fenologia de Virola sebifera Aubl. (Myristicaceae) em mata mesofítica de Uberlândia, MG, Brasil. Revista Brasileira de Botânica, São Paulo, v. 29, n. 3, p. 443-451, 2006.

LENZA, E.; OLIVEIRA, P. E. Biologia reprodutiva de Tapirira guianensis Aubl. (Anacardiaceae), uma espécie dioica em mata de galeria do Triângulo Mineiro, Brasil. Revista Brasileira de Botânica, São Paulo, v. 28, n. 1, p. 179-190, 2005.

LORENZI, H. Árvores brasileiras: manual de identificação e cultivo de plantas arbóreas nativas do Brasil. Nova Odessa: Plantarum, 1998. 368 p.

MORAES NETO, S. P. Árvores nativas do cerrado com potencial madeireiro. 2008. Disponível em: < http:// www.cpac.embrapa.br/ >. Acesso em: 31 jan. 2014.

OPLER, P. A.; BAWA, K. S. Sex ratios in tropical forest trees. Evolution, West Sussex, v. 32, n. 4, p. 812-821, 1978.

OTSUbO, H. C. B.; MORAES, M. L. T.; MORAES, M. A.; JOSÉ NETO, M.; FREITAS, M. L. M.; COSTA, R. B.; RESENDE, M. D. V.; SEBBENN, A. M. Variação genética para caracteres silviculturais em três espécies arbóreas da região do bolsão sul-mato-grossense. Cerne, Lavras, v. 21, n. 4, p. 535-544. 2015.

PALUDO, G. F.; MANTOVANI, A.; KLAUBERG, C.; REIS, M. S. Estrutura demográfica e padrão espacial de uma população natural de Araucaria angustifolia (Bertol.) Kuntze (araucariaceae), na Reserva Genética Florestal de Caçador, Estado de Santa Catarina. Revista Árvore, Viçosa, v. 33, n. 6, p. 1109-1121, 2009. PIMENTELGOMES, F.; GARCIA, C. H. Estatística aplicada a experimentos agronômicos e florestais: exposição com exemplos e orientações para uso de aplicativos. Piracicaba: FEALQ, 2002. 309 p.

RESENDE, M. D. V. Matemática e estatística na análise de experimento e no melhoramento genético. Colombo: Embrapa Florestas, 2007. 362 p.

RESENDE, M. D. V. Software selegen - REML/BLUP. Colombo: Embrapa Florestas, 2002a. 67 p. 
RESENDE, M. D. V. Genética biométrica e estatística no melhoramento de plantas perenes. Brasília: Embrapa Informação Tecnológica, 2002b. 975 p.

SANTOS, R. M.; VIEIRA, F. A.; M. FAGUNDES; NUNES, Y. R. F.; GUSMÃO, E. Riqueza e similaridade florística de oito remanescentes florestais no norte de Minas Gerais, Brasil. Revista Árvore, Viçosa, v. 31, n. 1, p. 135$144,2007$.

SEBBENN, A. M.; FREITAS, M. L. M.; ZANATTO, A. C. S.; MORAES, E. Seleção dentro de progênies de polinização aberta de Cariniana legalis Mart. O. Ktze (Lecythidaceae), visando à produção de sementes para recuperação ambiental. Revista Instituto Florestal, São Paulo, v. 21, n. 1, p. 27-37, 2009a.

SEBBENN, A. M.; FREITAS, M. L. M.; ZANATTO, A. C. S.; MORAES, E.; MORAES, M. A. Comportamento da variação genética entre e dentro de procedências e progênies de Gallesia integrifolia Vell. Moq. para caracteres quantitativos. Revista Instituto Florestal, São Paulo, v. 21, n. 2, p. 151-163, 2009 b.

SEBBENN, A. M.; FREITAS, M. L. M.; ZANATTO, A. C. S.; MORAES, E.; MORAES, M. A. Conservação ex situ e pomar de sementes em banco de germoplasma de Balfourodendron riedelianum. Revista Instituto Florestal, São Paulo, v. 19, n. 2, p. 101-112, 2007.

SEBBENN, A. M.; SIQUEIRA, A. C. M. F.; KAGEYAMA, P. Y.; MACHADO, J. A. R. Parâmetros genéticos na conservação da cabreúva - Myroxylon peruiferum L.F. Allemão. Scientia Forestalis, Piracicaba, n. 53, p. 31-38, 1998.

SIQUEIRA, A. C. M. F.; NOGUEIRA, J. C. B.; KAGEYAMA, P. Y. Conservação dos recursos genéticos ex situ do cambaru (Dipteryx alata) Vog. - Leguminosae. Revista Instituto Florestal, São Paulo, v. 5, n. 2, p. 231-243, 1993.

SOUZA, C. S.; AGUIAR, A. V.; SILVA, A. M.; MORAES, M. L. T. Variação genética em progênies de Gonçaloalves (Astronium fraxinifolium) em dois sistemas de plantio. Revista Instituto Florestal, São Paulo, v. 15, n. 2, p. 137-145, 2003.

VENCOVSKY, R. Tamanho efetivo populacional na coleta e preservação de germoplasma de espécies alógamas. Revista IPEF, Piracicaba, v. 35, p. 79-84, 1987.

VENCOVSKY, R.; CHAVES, L. J.; CROSSA, J. Variance effective size for dioecious species. Crop Science, Madison, v. 52, n. 1, p. 7990, 2012.

Recebido em 29/10/2016

Aceito para publicação em 13/04/2017

Sci. For., Piracicaba, v. 45, n. 115, p. 581-591, set. 2017 DOI: dx.doi.org/10.18671/scifor.v45n115.15 
